\title{
The Synergic Effects of Mirror Therapy and Neuromuscular Electrical Stimulation for Hand Function in Stroke Patients
}

\author{
Gi Jeong Yun, M.D., Min Ho Chun, M.D., Ji Young Park, M.D. ', Bo Ryun Kim, M.D.
}

Department of Rehabilitation Medicine, Asan Medical Center, University of Ulsan College of Medicine, Seoul 138-736, ${ }^{1}$ Phillip Hospital, Seoul 138-170, ${ }^{2}$ University of Jeju National University Hospital, Jeju 690-756, Korea

Objective To investigate the synergic effects of mirror therapy and neuromuscular electrical stimulation (NMES) for hand function in stroke patients.

Method Sixty patients with hemiparesis after stroke were included (41 males and 19 females, average age 63.3 years). Twenty patients had NMES applied and simultaneously underwent mirror therapy. Twenty patients had NMES applied only, and twenty patients underwent mirror therapy only. Each treatment was done five days per week, 30 minutes per day, for three weeks. NMES was applied on the surface of the extensor digitorum communis and extensor pollicis brevis for open-hand motion. Muscle tone, Fugl-Meyer assessment, and power of wrist and hand were evaluated before and after treatment.

Results There were significant improvements in the Fugl-Meyer assessment score in the wrist, hand and coordination, as well as power of wrist and hand in all groups after treatment. The mirror and NMES group showed significant improvements in the Fugl-Meyer scores of hand, wrist, coordination and power of hand extension compared to the other groups. However, the power of hand flexion, wrist flexion, and wrist extension showed no significant differences among the three groups. Muscle tone also showed no significant differences in the three groups.

Conclusion Our results showed that there is a synergic effect of mirror therapy and NMES on hand function. Therefore, a hand rehabilitation strategy combined with NMES and mirror therapy may be more helpful for improving hand function in stroke patients than NMES or mirror therapy only.

Key Words Mirror therapy, Neuromuscular electrical stimulation, Stroke

\section{INTRODUCTION}

Received July 28, 2010; Accepted November 2, 2010

Corresponding author: Min Ho Chun

Department of Rehabilitation Medicine, Asan Medical Center, University of Ulsan College of Medicine, 388-1, Pungnap-2 dong, Songpa-gu, Seoul 138-736, Korea

Tel: +82-2-3010-3800, Fax: +82-2-3010-6964, E-mail: mhchun@amc. seoul.kr

Copyright $\odot 2011$ by Korean Academy of Rehabilitation Medicine
Impairment of motor function is common in stroke patients and handicaps them to conduct activities of daily life. It is known that $87 \%$ of motor impairments of the upper extremities occur in the acute stage ${ }^{1}$ and in many cases the continued motor impairment of upper extremities restrict activities of daily life. Various treatment techniques or therapies have been employed 
to improve impaired motor functions of the upper extremity, but they are usually focused on paretic arms. Additionally, they require one-on-one treatment with therapists, which means that the effort and time of a therapist are absolutely essential. ${ }^{2}$ On the other hand, mirror therapy and neuromuscular electrical stimulation (NMES) are simple and less costly, and patients can perform these by themselves. Mirror therapy was first described by Ramachandran and Rogers-Ramachandran. They reported the reduction of pain when using visual ${ }^{3}$ illusion for phantom pain. Previous studies showed that mirror therapy improved the motor function of upper extremities. ${ }^{4,5}$ Recently, Stevens et al. ${ }^{6}$ reported that mirror therapy made significant improvements in the Brunnstrom stage as well as functional independence measure scores of subacute stroke patients. NMES makes improvements in the motor function of patients with hemiparesis such as reduced spasticity, strengthened muscles, and increased motion range of joints. ${ }^{7}$ Thus, the study examined the combination of mirror therapy and NMES, and determined whether they result in significant improvement of recovering the motor function of upper extremities in hemiparetic patients.

\section{MATERIALS AND METHODS}

\section{Subjects}

The study was conducted on 60 patients who were admitted or transferred to the Department of Rehabilitation at the medical center due to hemiparesis caused by stroke between March 1, 2009 and March 30, 2010. The studies excluded those who were expected to be uncooperative due to cognitive impairment, were medically unstable, and had neurologic deficit, or patients with neglect. The subjects were randomly assigned with the random number table to the mirror therapy and NMES group (20 persons), the mirror therapy only group (20 persons) or the NMES only group (20 persons).

\section{Method}

All three groups received the same conventional rehabilitation programs and additionally, had each of their own therapies for thirty minutes, five days a week for three weeks. For the mirror therapy group, with their paretic arm put behind the mirror, they kept bending and extending the nonparetic-side wrist and hand while patients looked into the mirror watching the movements of their non-paretic hand and imagined their paretic-side wrist and hand were doing exactly the same thing. Electrical stimulation (Microstim ${ }^{\circledR}$ Model $\mathrm{GmbH}$, Stanberg, Germany) was applied at 30-70 mA intensity, $250 \mu \mathrm{sec}$ amplitude, and $35 \mathrm{~Hz}$ frequency. It lasted for five seconds and then stopped for five seconds. The intensity of stimulation was determined so that the subjects could feel muscle contraction while not feeling tired. It was applied to the common extensor digitorum muscle and extensor polliics brevis of the paretic arm with an aim at hand extension movements. ${ }^{7}$ At the same time, patients actively practiced paretic-side wrist and hand flexion and extension to electrical stimuli. For the mirror and NMES therapy group and NMES only group, patients extended their paretic wrists and hands and at the same time extended non-paretic wrists and hands to electrical stimuli. They also actively conducted nonparetic wrist and hand flexion when bending the paretic wrist and hand with their paretic wrist and hand not extended, which was caused by absence of electrical stimuli. The NMES therapy group looked into an opaque wooden board while conducting the same thing as the mirror and NMES therapy group did. The mirror therapy group repeated bending and extending their paretic wrists and hands at an interval of five seconds while looking into the mirror when they were conducting flexion and extension movements of non-paretic wrists and hands. For example, as shown in Fig. 1-A, the patient with right hemiparesis had NMES on her right wrist and hand extensor muscle and simultaneously underwent flexion and extension of her fingers and wrist while looking at the reflection of her left hand on the mirror. Fig. 1-B (the mirror therapy group) shows the patient with right hemiparesis underwent flexion and extension of hand and wrist while looking at the reflection on the mirror. Fig. 1-C (NMES group) shows a patient with right hemiparesis had NMES on the right wrist and hand extensor muscle and at the same time, underwent flexion and extension of hand and wrist while looking at the wooden board (Fig. 1). Fugl-Meyer assessment was conducted on all subjects to evaluate the motor functions of the upper extremity before and after treatment and the power of wrist and hand was measured in the manual muscle test. Severity or score of spasticity of 

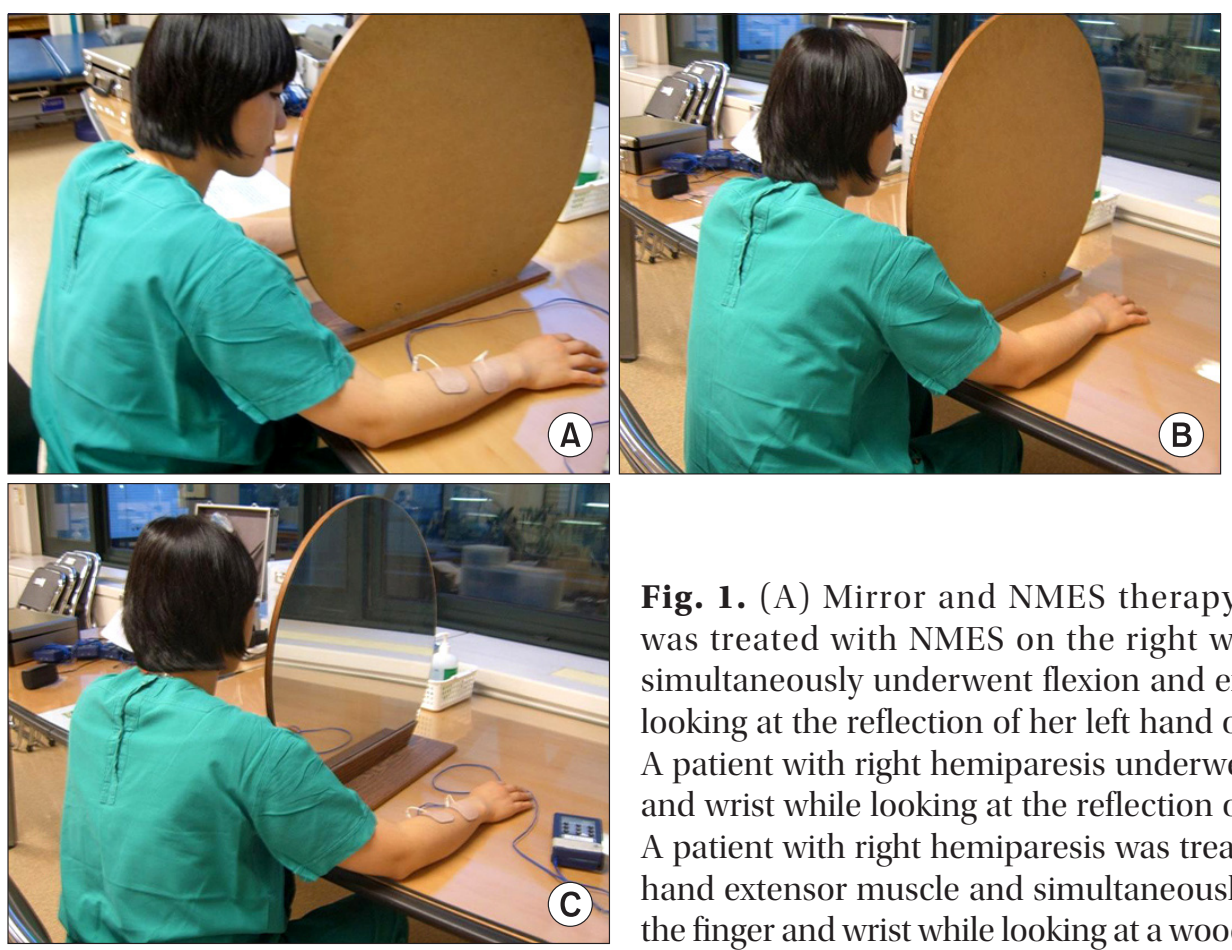

Fig. 1. (A) Mirror and NMES therapy: A patient with right hemiparesis was treated with NMES on the right wrist and hand extensor muscle and simultaneously underwent flexion and extension of the finger and wrist while looking at the reflection of her left hand on the mirror. (B) Mirror therapy only: A patient with right hemiparesis underwent flexion and extension of the finger and wrist while looking at the reflection on the mirror. (C) NMES therapy only: A patient with right hemiparesis was treated with NMES on the right wrist and hand extensor muscle and simultaneously underwent flexion and extension of the finger and wrist while looking at a wooden board.

hand extensor muscle was quantified using the modified Ashworth scale.

\section{Statistical analysis}

The data was statistically analyzed using SPSS 14.0 version. The mean and standard deviation of the data were obtained through descriptive statistics. The comparison of the three groups before and after treatment was made using the Wilcoxon test. The Kruskal-Wallis test was used to measure improvements of motor functionality of upper extremity and the MannWhitney Test was used for post hoc test. The level of significance (p-value) was Bonferroni corrected. Statistical significance ( $\mathrm{p}$-value) was set at $\mathrm{p}<0.05$.

\section{RESULTS}

\section{Demographic characteristics of patients}

The mirror and NMES therapy group consisted of twelve males and eight females, the mirror therapy group of fourteen males and six females and the NMES group of thirteen males and seven females. Overall, there were more male subjects than females. The mirror and NMES therapy group was $65.9 \pm 10.8$ years old on average, whereas the mirror therapy group $63.1 \pm 7.3$ years old. The last NMES group was $61.0 \pm 8.4$ years old on average. Even though it was older than the other two groups, there was no significant difference among the three groups. Brain lesions appeared similar in the left and right sides. There were 16 patients with cerebral infarction and 4 patients with cerebral hemorrhage in the mirror and NMES therapy group, respectively. The mirror therapy group had 15 patients with cerebral infarction and 5 patients with cerebral hemorrhage, whereas the NMES group had 15 patients with cerebral infarction and 5 patients with cerebral hemorrhage. There were many more patients with cerebral infarction than those with cerebral hemorrhage in all the groups. It took $25.8 \pm 12.6$ days for the groups from the onset of stroke to treatment. In the Korean version of mini-mental state examination, the mirror and NMES therapy group scored $23.0 \pm 4$.6 points, the mirror therapy group $22.5 \pm 4.4$ points and the NMES group 22.1 \pm 3.4 points (Table 1 ).

\section{Effect before and after treatment}

All the three groups showed significant improvements in power of flexion and extension of wrists and hands after treatment. They showed significant improvements in the Fugl-Meyer scores regarding the wrist, hand and coordination items, both respectively and combined. 
Although the three groups showed a slight increase in spasticity, these findings were not statistically significant (Table 2).

\section{Comparison of post-treatment effects in three groups}

When effects before and after treatment were compared

Table 1. Demographic Characteristics of the Patients in This Study

\begin{tabular}{|c|c|c|c|}
\hline & $\begin{array}{c}\text { Mirror + } \\
\text { NMES }\end{array}$ & Mirror & NMES \\
\hline Number & 20 & 20 & 20 \\
\hline $\begin{array}{l}\text { Gender } \\
\text { (male : female) }\end{array}$ & $12: 8$ & $14: 6$ & $13: 7$ \\
\hline Age (years) & $65.9 \pm 10.8$ & $63.1 \pm 7.3$ & $61.0 \pm 8.4$ \\
\hline $\begin{array}{l}\text { Brain lesion } \\
\text { (right: left) }\end{array}$ & $10: 10$ & $9: 11$ & $12: 8$ \\
\hline $\begin{array}{l}\text { Infarction: } \\
\text { Hemorrhage }\end{array}$ & $16: 4$ & $15: 5$ & $15: 5$ \\
\hline \multicolumn{4}{|l|}{ Location } \\
\hline MCA & 15 & 15 & 16 \\
\hline Brain stem & 2 & 3 & 3 \\
\hline Multiple & 3 & 2 & 1 \\
\hline $\begin{array}{l}\text { Time post stroke } \\
\text { (days) }\end{array}$ & $25.6 \pm 14.4$ & $23.9 \pm 10.5$ & $28.1 \pm 12.8$ \\
\hline K-MMSE & $23.0 \pm 4.6$ & $22.5 \pm 4.4$ & $22.1 \pm 3.4$ \\
\hline
\end{tabular}

MCA: Middle cerebral artery, K-MMSE: Korean version of mini-mental state examination, NMES: Neuromuscular electrical stimulation for the groups, the hand extension power, and both respective Fugl-Meyer scores of wrist, hand and coordination and combined scores were significantly higher in the mirror and NMES therapy group than the other two groups. There was no significant improvement in spasticity, wrist power and hand power (Table 3).

\section{DISCUSSION}

This study showed significant improvements in wrist and hand power when the mirror therapy was applied in combination with NMES therapy. The NMES group showed some improvements in motor functions compared with the mirror therapy group, but this was not considered statistically significant. The three groups did not show a significant difference in spasticity.

Several mechanisms have been suggested to explain how mirror therapy works for motor recovery. Alschuler et al. ${ }^{4}$ proposed that the mirror reflection of the goodmoving arm, which looks like the affected arm moving correctly, substitutes the often decreased or void proprioceptive input, thus helping recruit the premotor cortex and improve motor rehabilitation through close interaction with the premotor cortex. Stevens and Stoykov ${ }^{5}$ hypothesized that congruent visual feedback from the moving nonparetic hand, as provided by a mirror, would restore the function of the affected hand. Garry et al. ${ }^{8}$ performed transcranial magnetic stimulation during mirror illusion in healthy subjects and showed

Table 2. Improvement of Clinical Parameters in Three Groups after 3 Weeks Treatment

\begin{tabular}{lcccccc}
\hline & \multicolumn{2}{c}{ Mirror + NMES } & \multicolumn{2}{c}{ Mirror } & \multicolumn{2}{c}{ NMES } \\
\cline { 2 - 7 } & Before & After & Before & After & Before & After \\
\hline MAS & $0.4 \pm 0.5$ & $0.7 \pm 0.5$ & $0.2 \pm 0.4$ & $0.7 \pm 0.5$ & $0.4 \pm 0.5$ & $0.8 \pm 0.8$ \\
HF & $1.2 \pm 0.8$ & $2.8 \pm 1.1^{*}$ & $1.3 \pm 1.0$ & $2.4 \pm 1.2^{*}$ & $1.2 \pm 0.6$ & $2.5 \pm 0.7^{*}$ \\
HE & $1.4 \pm 1.0$ & $3.0 \pm 1.0^{*}$ & $1.8 \pm 1.0$ & $2.6 \pm 1.0^{*}$ & $1.7 \pm 0.9$ & $2.6 \pm 0.5^{*}$ \\
WF & $1.2 \pm 0.8$ & $2.8 \pm 1.1^{*}$ & $1.3 \pm 1.0$ & $2.3 \pm 1.2^{*}$ & $1.2 \pm 0.6$ & $2.5 \pm 0.7^{*}$ \\
WE & $1.4 \pm 1.0$ & $3.0 \pm 1.0^{*}$ & $1.8 \pm 1.0$ & $2.6 \pm 1.0^{*}$ & $1.8 \pm 0.9$ & $2.8 \pm 0.4^{*}$ \\
FMW & $1.6 \pm 1.9$ & $8.7 \pm 1.8^{*}$ & $2.1 \pm 2.2$ & $4.3 \pm 1.7^{*}$ & $2.0 \pm 1.8$ & $4.7 \pm 1.8^{*}$ \\
FMH & $2.0 \pm 2.5$ & $9.0 \pm 3.3^{*}$ & $2.4 \pm 2.5$ & $5.2 \pm 3.8^{*}$ & $2.3 \pm 1.7$ & $7.7 \pm 2.7^{*}$ \\
FMC & $0.8 \pm 1.3$ & $3.0 \pm 1.3^{*}$ & $1.0 \pm 1.3$ & $1.9 \pm 1.3^{*}$ & $1.0 \pm 0.6$ & $2.8 \pm 1.7^{*}$ \\
FMS & $4.3 \pm 5.3$ & $20.7 \pm 5.1^{*}$ & $5.3 \pm 5.8$ & $11.2 \pm 6.9^{*}$ & $5.3 \pm 3.3$ & $15.3 \pm 6.9^{*}$ \\
\hline
\end{tabular}

MAS: Modified Ashworth scale, HF: Power of hand flexion, HE: Power of hand extension, WF: Power of wrist flexion, WE: Power of wrist extension, FMW: Fugl-Meyer score of wrist, FMH: Fugl-Meyer score of hand, FMC: Fugl-Meyer score of coordination, FMS: Summation of Fugl-Meyer score of wrist, hand and coordination ${ }^{*} \mathrm{p}<0.05$ 
Table 3. Comparison of Improvement after 3 Weeks Treatment in Each Group

\begin{tabular}{lccc}
\hline & Mirror+NMES & Mirror & NMES \\
\hline$\Delta$ MAS & $0.3 \pm 0.8$ & $0.5 \pm 0.7$ & $0.4 \pm 0.5$ \\
$\Delta$ Wrist F & $1.6 \pm 0.9$ & $1.1 \pm 0.7$ & $1.3 \pm 0.7$ \\
\hline$\Delta$ Wrist E & $1.6 \pm 1.2$ & $0.8 \pm 0.4$ & $1.0 \pm 0.8$ \\
$\Delta$ Hand F & $1.6 \pm 0.9$ & $1.1 \pm 0.7$ & $1.3 \pm 0.7$ \\
$\Delta$ Hand E & $1.6 \pm 1.2^{*}$ & $0.8 \pm 0.4$ & $0.9 \pm 0.7$ \\
$\Delta$ FMW & $7.1 \pm 2.3^{*}$ & $2.3 \pm 1.2$ & $2.7 \pm 1.8$ \\
$\Delta$ FMH & $6.8 \pm 3.4^{*}$ & $3.4 \pm 0.8$ & $5.5 \pm 2.1$ \\
$\Delta$ FMC & $2.3 \pm 1.3^{*}$ & $1.0 \pm 0.9$ & $1.8 \pm 1.1$ \\
\hline FMS & $16.2 \pm 5.8^{*}$ & $6.2 \pm 3.4$ & $10.0 \pm 0.3$ \\
\hline
\end{tabular}

MAS: Modified Ashworth scale, HF: Hand F, HE: Hand E, WF: Wrist F, WE: Wrist E, FMW: Fugl-Meyer score of wrist, FMH: Fugl-Meyer score of hand, FMC: Fugl-Meyer score of coordination, FMS: Summation of Fugl-Meyer score of wrist, hand and coordination ${ }^{*} \mathrm{p}<0.05$

increased excitability of the primary motor cortex of the hand behind the mirror. In addition, Liepert et al. ${ }^{10}$ reported that the primary motor cortex was excited by hand movements and thus the ipsilateral MI excitability is known to increase contraction strength as voluntary unilateral arm/hand movements induced excitability changes in both the contralateral and ipsilateral primary motor cortex (M1). ${ }^{9}$

The NMES study showed NMES activated bilateral motor and sensory cortex on healthy subjects, ${ }^{11-13}$ and concluded in the meta-analysis that NMES improved motor recovery of upper extremities in stroke patients. ${ }^{14}$ The electrical stimulation, however, was reported to induce the most meaningful improvements in patients with mild to moderate degree of hand handicap. ${ }^{10,11,15}$ Santos et al. ${ }^{16}$ reported that when ten times higher electrical stimulation was applied to chronic severe stoke patients, there was significant post-treatment improvement of motor functions in both groups. One group was told to do flexion and extension of hands and wrists to electrical stimuli, and the other group was instructed to reach out, grab and move an object through the electrical stimulation. The latter group showed more significant improvements than the former. They concluded that the combination of electrical stimulation with active movements would help improve motor recovery. In this study, fifteen times higher electrical stimulation was applied to subacute stage stroke patients and raised compliance. The electrical stimuli were enough to induce any improvement in motor recovery. The three groups showed significant improvements in motor functions of wrists and hands after treatment. It seems that the therapeutic effect is combined with recovery with time elapse after stroke. When compared among three groups before and after treatment, there was no significant difference in wrist power and hand flexion power, but the mirror and NMES therapy group showed more improvement in hand extension power and a significant increase in Fugl-Meyer scores. This proves previous studies that found active movement of a patient had positive effects on motor function rather than electrical stimulation alone. In particular, the reason for statistically significant improvement in hand extension power is that an electrical stimulation was not applied to hand flexor muscle in the case of hand flexion, while it was applied to hand and wrist extensor muscle in the case of hand extension, when both mirror and NMES therapies were used. This study was conducted on subacute stroke patients, so there were many patients with severe motor impairment. Even though several patients showed improvements in motor functions after treatment, they still had severe motor impairment. Therefore, it was difficult to obtain therapeutic effects. Based on previous studies reporting that patients with mild to moderate severity of motor impairment were conducive to the treatment, ${ }^{9,11,15}$ improvements would have been much more conspicuous through more upper extremity motor function assessment methods if the treatment had been applied to patients who began to show movements of upper extremities. For patients whose senses were more numb, therapists or guardians had to keep stimulating them with electrical stimuli to carry out the mirror and NMES therapies at the same time, so the patients could not do the treatment themselves. This is likely to affect treatment results. In addition, subscores of Fugl-Meyer Scale were analyzed respectively, but the entire scores were not analyzed in the upper extremity motor function assessment, which indicates the assessment method has its own limitation. Both the mirror and NMES therapies seem to improve motor functions by affecting the activation of the brain in the primary motor cortex. Also this study took only functional improvements into 
account. Therefore, it could not determine whether the simultaneous application of the mirror and NMES therapies affected the activation of the brain or if stronger activation of brain resulted in improved motor functions. Further studies need to elucidate if both therapies made synergic effects in brain activation.

\section{CONCLUSION}

This study aimed to examine the simultaneous application of the mirror and NMES therapies, which could improve the recovery of upper extremity motor function for stroke patients. These therapies are not dependent upon therapists, so they can be applied at home after patients have been discharged from a hospital. Therefore, the simultaneous application of the mirror therapy and NMES therapy for subacute stroke patients with hemiparesis must be considered as one of the rehabilitation programs to improve upper extremity motor functions of stroke patients.

\section{REFERENCES}

1. Parker VM, Wade DT, Langton Hewer R. Loss of arm function after stroke: measurement, frequency, and recovery. Int Rehabil Med 1986; 8: 69-73

2. Prange GB, Jannink MJ, Groothuis-Oudshoorn CG, Hermens HJ, Ijzerman MJ. Systematic review of the effect of robot-aided therapy on recovery of the hemiparetic arm after stroke. J Rehabil Res Dev 2006; 43: 171-184

3. Ramachandran VS, Rogers-Ramachandran D. Synaesthesia in phantom limbs induced with mirrors. Proc Biol Sci 1996; 263: 377-386

4. Altschuler EL, Wisdom SB, Stone L, Foster C, Galasko D, Llewellyn DM, Ramachandran VS. Rehabilitation of hemiparesis after stroke with a mirror. Lancet 1999; 353: 2035-2036

5. Stevens JA, Stoykov ME. Using motor imagery in the rehabilitation of hemiparesis. Arch Phys Med Rehabil 2003; 84: 1090-1092

6. Yavuzer G, Selles R, Sezer N, Sutbeyaz S, Bussmann JB, Koseoglu F, Atay MB, Stam HJ. Mirror therapy improves hand function in subacute stroke: a rando- mized controlled trial. Arch Phys Med Rehabil 2008; 89: 393-398

7. Han TR, Lim SJ, Kim DY, Lee KJ. Stimulation intensity of functional electrical stimulation on hemiplegic upper extremity. J Korean Acad Rehab Med 2002; 26: 379-384

8. Garry MI, Loftus A, Summers JJ. Mirror, mirror on the wall: viewing a mirror reflection of unilateral hand movements facilitates ipsilateral M1 excitability. Exp Brain Res 2005; 163: 118-122

9. Liepert J, Dettmers C, Terborg C, Weiller C. Inhibition of ipsilateral motor cortex during phasic generation of low force. Clin Neurophysiol 2001; 112: 114-121

10. Kimberley TJ, Lewis SM, Auerbach EJ, Dorsey LL, Lojovich JM, Carey JR. Electrical stimulation driving functional improvements and cortical changes in subjects with stroke. Exp Brain Res 2004; 154: 450-460

11. Wu CW, Seo HJ, Cohen LG. Influence of electric somatosensory stimulation on paretic-hand function in chronic stroke. Arch Phys Med Rehabil 2006; 87: 351-357

12. Wu CW, van Gelderen P, Hanakawa T, Yaseen Z, Cohen LG. Enduring representational plasticity after somatosensory stimulation. Neuroimage 2005; 27 : 872-884

13. Conforto AB, Kaelin-Lang A, Cohen LG. Increase in hand muscle strength of stroke patients after somatosensory stimulation. Ann Neurol 2002; 51: 122125

14. de Kroon JR, van der Lee JH, IJzerman MJ, Lankhorst GJ. Therapeutic electrical stimulation to improve motor control and functional abilities of the upper extremity after stroke: a systematic review. Clin Rehabil 2002; 16: 350-360

15. Cauraugh J, Light K, Kim S, Thigpen M, Behrman A. Chronic motor dysfunction after stroke: recovering wrist and finger extension by electromyographytriggered neuromuscular stimulation. Stroke 2000; 31: 1360-1364

16. Santos M, Zahner LH, McKiernan BJ, Mahnken JD, Quaney B. Neuromuscular electrical stimulation improves severe hand dysfunction for individuals with chronic stroke: a pilot study. J Neurol Phys Ther 2006; 30: 175-183 